\title{
ACKNOWLEDGMENT OF REVIEWERS
}

The success of Molecular Plant-Microbe Interactions depends on the quality of manuscripts submitted by authors and on the care and competence with which they are reviewed. It is the policy of the Editorial Board to solicit reviews of manuscripts from specialists most qualified to review them. In addition to members of the Editorial Board, the individuals listed below provided constructive critical reviews of one or more manuscripts during the past year. Their names are published here in grateful appreciation for their contributions to the journal.

P. Albersheim, University of Georgia, Athens, GA, U.S.A.

A Alvares-Morales, Cinvestav IPN - Irapuato, Mexico

A. Anderson, Utah State University, Logan, UT, U.S.A.

C. J. Baker, USDA, MPPL, BARC-WEST, Beltsville, MD, U.S.A.

J. Bakker, Wageningen Agricultural University, Wageningen, The Netherlands

H. Barker, Scottish Crop Research Institute, Invergowrie, Dundee, Scotland, United Kingdom

D. Beck, The Horticulture and Food Research Institute of New Zealand Ltd., Auckland, New Zealand

A. Becker, University Bielefeld, Bielefeld, Germany

S. V. Beer, Cornell University, Ithaca, New York, U.S.A.

A. Bent, University of Wisconsin-Madison, Madison, WI, U.S.A.

C. Bergman, University of Georgia, Athens, GA, U.S.A.

P. R. Birch, Scottish Crop Research Institute, Invergowie, Dundee, Scotland, United Kingdom

D. M. Bird, North Carolina State University, Raleigh, NC, U.S.A.

T. Bisseling, Wageningen Agricultural University, Wageningen, The Netherlands

G. Bloemberg, Leiden University, Leiden, The Netherlands

M. Boccara, Istituto di Fitovirologia Applicata, Torino, Italy

U. Bonas, Martin-Luther University. Halle (Salle), Germany

P. Bonfante, Universita Torino, Torino, Italy

R. Bostock, University of California, Davis, CA, U.S.A.

D. C. Boyes, Paradigm Genetics, Inc., Research Triangle Park, NC, U.S.A.

C. Brasier, Alice Holt Lodge, Surrey, United Kingdom

C. Bronson, Iowa State University, Ames, IA, U.S.A.

W. Broughton, Universite de Geneve, Geneve, Switzerland

K. W. Buck, Imperial College of Science, London, United Kingdom

B. Bushnell, University of Minnesota, St. Paul, MN, U.S.A.

M. Camacho, Leiden University, Leiden, The Netherlands

J. C. Carrington, Washington State University, Pullman, WA, U.S.A.

C. E. Caten, University of Birmingham, Birmingham, United Kingdom

J. Chappell, University of Kentucky, Lexington, KY, U.S.A.

A Chatterjee, University of Missouri, Columbia, MO, U.S.A.

Z. Chen, University of Idaho, Moscow, ID, U.S.A.

J. Chittoor, Monsanto Company. St. Louis, MO, U.S.A.

A. Collmer, Cornell University, Ithaca, NY, U.S.A.

G. Condemine, CNRS URA 1486, Villeurbanne, France

I. Connerton, University of Nottingham, Loughborough, United Kingdom

J. E. Cooper, Queens University Belfast, Northern Ireland, United Kingdom

D. Coplin, Ohio State University, Columbus, OH, U.S.A.
S. Covert, University of Georgia, Athens, GA, U.S.A.

J. Culver, University of Maryland, College Park, MD, U.S.A.

E. L. Davis, North Carolina State University, Raleigh, NC, U.S.A.

K. Davis, Paradigm Genetics, Inc. Research Triangle Park, NC, U.S.A.

L. Davis, Kansas State University, Manhattan, KS, U.S.A.

F. J. de Bruijn, Michigan State University, East Lansing, MI, U.S.A.

R. Dean, Clemson University, Clemson, SC, U.S.A.

F. Debelle, INRA-CNRS, Castanet-Tolosan, France

T. Denny, University of Georgia, Athens, Georgia, U.S.A.

Y. Dessaux, ISV-CNRS, Gif-sur-Yvette, France

P. J. G. M. De Wit, Wageningen Agricultural University, Wageningen, The Netherlands

M. B. Dickman, University of Nebraska, Lincoln, NE, U.S.A.

B. Dietrich, Novartis Crop Protection, Research Triangle Park, NC, U.S.A.

R. Dixon, John Innes Centre, Norwich, United Kingdom

M. Djordjevic, The Australian National University, Canberra City, Australia

X. Dong, Duke University, Durham, NC, U.S.A.

J. Doonan, John Innes Centre, Norwich, United Kingdom

J. M. Dow, John Innes Centre, Norwich, United Kingdom

J. A. Downie, John Innes Centre, Norwich, United Kingdom

J. Dubcovsky, University of California, Davis, CA, U.S.A.

M. Dufresne, Centre de Recherche sur les Plantes, Orsay, France

I. Dusha, Institute of Genetics. Szeged, Hungary

J. Duvick, Pioneer Hi-Bred, Johnston, IA, U.S.A.

J. R. Ecker, The University of Pennsylvania, Philadelphia, PA, U.S.A.

P. Epple, University of North Carolina, Chapel Hill, NC, U.S.A.

D. Expert, INA P-G/INRA, Paris, France

M. L. Farman, University of Kentucky, Lexington, KY, U.S.A.

S. Farrand, University of Illinois at Urbana-Champaign, Urbana, IL, U.S.A.

G. Felix, Friedrich Miescher-Institut, Basel, Switzerland

H. Felle, Universitat Giessen, Giessen, Germany

T. Finan, McMaster University, Hamilton, Ontario, Canada

P. Franken, Max-Planck-Institut fur Terrestriche Mikrobiologie, Marburg, Germany

H. Franssen, Wageningen Agricultural University, Wageningen, The Netherlands

T. Gaffney, Novartis Agribusiness Biotechnology Research, Research Triangle Park, NC, U.S.A.

P. Gamas, INRA-CNRS, Castanet-Tolosan, France

J. AntonioGarcia, Campus de la Universidad Autonoma de Madrid, Madrid, Spain 
M. Garnier, INRA, Villenave d'Ornon, France

V. Gianinazzi-Pearson, INRA-CNRS, Dijon, France

H. Giese, Riso Nat'1 Lab, Roskilde, Denmark

R. Gilbertson, University of California-Davis, Davis, CA, U.S.A.

D. Gilchrist, University of California-Davis, Davis, CA, U.S.A.

L. Glover, University of Aberdeen, Aberdeen, Scotland, United Kingdom

R. Goldbach, Wageningen Agricultural University, Wageningen, The Netherlands

M. Grant, Wye College, Wye, Ashford, Kent, United Kingdom

S. Gray, Cornell University, Ithaca, NY, U.S.A.

D. Gross, Washington State University, Pullman, WA, U.S.A.

E. Grotewold, The Ohio State University. Columbus, $\mathrm{OH}$, U.S.A.

M. Hahn, Universitaet Konstanz, Konstanz, Germany

R. Hammerschmidt, Michigan State University E. Lansing, MI, U.S.A.

J. Handelsman, University of Wisconsin-Madison, Madison, WI, U.S.A.

M. J. Harrison, Samuel Roberts Noble Foundation, Ardmore, OK, U.S.A.

S. Y. He, Michigan State University E. Lansing, MI, U.S.A.

M. Heath, University of Toronto. Toronto, Ontario, Canada

J. P. Helgeson, University of Wisconsin-Madison, Madison, WI, U.S.A.

E. Hilaire, Kansas State University, Manhattan, KS, U.S.A.

D. Hildebrand, University of Kentucky, Lexington, KY, U.S.A.

A. M. Hirsch, University of California, Los Angeles, CA, U.S.A.

T. Hohn, Novartis Seeds, Research Triangle Park, NC, U.S.A.

B. Holt, University of North Carolina, Chapel Hill, NC, U.S.A.

E. Holub, Horticulture Research International, Wellesbourne, Warwickshire, United Kingdom

S. Hulbert, Kansas State University, Manhattan, KS, U.S.A.

S. W. Hutcheson, University of Maryland, College Park, MD, U.S.A.

R. Innes, Indiana University, Bloomington, IN, U.S.A.

D. Inze, University of Gent, Gent, Belgium

T. Jabs, BASF AG AP/RF, Liomburgerhof, Germany

M. K. Jahn, Cornell University, Ithaca, NY, U.S.A.

D. Jeenes, John Innes Centre. Norwich, United Kingdom

G. Johal, DR Group, Johnston, IA, U.S.A.

H. Judelson, University of California, Riverside, CA, U.S.A.

R. Kahmann, Inst fur Genetik, Munchen, Germany

M. Kahn, Washington State University, Pullman, WA, U.S.A.

S. Kamoun, The Ohio State University, Wooster, OH, U.S.A.

P. Katinakis, Agricultural University of Athens, Athens, Greece

N. Keen, University of California, Riverside, CA, U.S.A.

G. Kema, DLO Research Institute for Plant Protection, Wageningen, The Netherlands

J. Kieber, University of North Carolina, Chapel Hill, NC, U.S.A.

J. Kijne, Leiden University, Leiden, The Netherlands

E. Kombrink, Max-Planck Institut fuer Zuechtungsforschung, Koln, Germany
A. Kondorosi, CNRS, Gif-Sur-Yvette, France

E. Kondorosi, CNRS, Gif-Sur-Yvette, France

K. Lambert, University of California, Berkeley, CA, U.S.A.

T. Langin, Universite Paris-Sud, Orsay, France

C. Lauriere, CNRS, Gif-Sur-Yvette, France

K. A. Lawton, Novartis Crop Protection, Inc., Research Triangle Park, NC, U.S.A.

A. Levine, The Hebrew University of Jerusalem, Jerusalem, Israel

Y. Li, University of Connecticut, Storrs, CT, U.S.A.

H. J. Linthorst, Leiden University, Leiden, The Netherlands

S. A. Lommel, North Carolina State University, Raleigh, NC, U.S.A.

J. Loper, USDA ARS, Corvallis, OR, U.S.A.

P. S. Low, Purdue University, West Lafayette, IN, U.S.A.

E. Lozoya, CINVESTAV-IPN, Irapuato, Guanjuato, Mexico

B. J. J. Lugtenberg, Leiden University, Leiden, The Netherlands

G. Lyon, Scottish Crop Research Institute. Invergowie, Dundee, Scotland, United Kingdom

K. Maleck, Novartis Biotechnology, Research Triangle Park, NC, U.S.A.

R. Malmberg, University of Georgia, Athens, GA, U.S.A.

J. M. Mansfield, Wye College (University of London), Wye, Ashford, Kent, United Kingdom

E. Marois, Martin-Luther Universitat, Halle (Salle), Germany

G. B. Martin, Boyce Thompson Institute at Cornell University, Ithaca, NY, U.S.A.

S. McCouch, Cornell University, Ithaca, NY, U.S.A.

J. McDowell, University of North Carolina, Chapel Hill, NC, U.S.A.

J. P. Metraux, University of Fribourg, Fribourg, Switzerland

R. W. Michelmore, University of California, Davis, CA, U.S.A.

A. Molina, E. T. S. Ingenieros Agronomos, Madrid, Spain

J. B. Morel, INRA, Versailles, France

J. F. Murphy, Auburn University, Auburn, AL, U.S.A.

U. Nehls, Botanisches Institut, Physiologische Okologie der Pflanzen, Tubingen, Germany

E. Nester, University of Washington, Seattle, W, U.S.A.

T. Niblack, University of Missouri, Columbia, MO, U.S.A.

K. Niehaus, Universitat Bielefeld, Bielefeld, Germany

Z. Nimchuk, University of North Carolina, Chapel Hill, NC, U.S.A.

T. Nuernberger, Institute of Plant Biochemistry, Halle(Salle), Germany

D. Nuss, University of Maryland, College Park, MD, U.S.A.

R. Oliver, Carlsberg Laboratory, Copenhagen Valby, Denmark

T. Palva, University of Helsinki, Helsinki, Finland

M. Parniske, John Innes Centre, Colney Lane, Norwich, United Kingdom

G. Payne, North Carolina State University, Raleigh, NC, U.S.A.

S. Perrotto, Istituto Universitario Navale, Napoli, Italy

B. Pfender, USDA-ARS NFSPRC, Corvallis, OR, U.S.A.

D. Phillips, University of California, Davis, CA, U.S.A.

L. S. Pierson, University of Arizona, Tucson, AZ, U.S.A.

C. M. J. Pieterse, Utrecht University, Uthrecht, The Netherlands

M. Pirhonen, Swedish University of Agricultural Sciences, Uppsala, Sweden 
A. Poplawsky, University of Idaho, Moscow, ID, U.S.A.

A. L. N. Rao, University of California, Riverside, CA, U.S.A.

R. W. Ridge, International Christian University, Mitaka-shi, Tokyo

I. Roberts, University of Manchester, Manchester, United Kingdom

D'A. Rochon, Agric. \& Agri-Food Canada, Summerland, B.C., Canada

T. Romeis, John Innes Institute, Norwich, Norfolk, United Kingdom

L. Rosendahl, Riso National Laboratory, Roskilde, Denmark

J. E. Ruiz Sainz, University de Sevilla, Sevilla, Spain

M. Sadowsky, University of Minnesota, St. Paul, MN, U.S.A.

S. Santa Cruz, Scottish Crop Research Institute, Invergowie, Dundee, Scotland, United Kingdom

G. Sarath, University of Nebraska-Lincoln, Lincoln, NE, U.S.A.

C. Sautter, Institute of Plant Sciences ETH, Zurich, Switzerland

H. Scholthof, Leiden University, Leiden, The Netherlands

J. Scott-Craig, Michigan State University, East Lansing, MI, U.S.A.

S. Seal, University of Greenwich, Kent, United Kingdom

R. Serrano, Universidad Politecnica de Valencia-C.S.I.C., Valencia, Spain

J. Shah, Kansas State University, Manhattan, KS, U.S.A.

J. G. Shaw, University of Kentucky, Lexington, KY, U.S.A.

H. Slater, John Innes Centre, Colney Lane, Norwich, United Kingdom

B. Solheim, University of Tromso, Tromso, Norway

I. E. Somssich, Max-Planck-Institut f. Zuechtungsforschung, Koeln, Germany

G. Stacey, University of Tennessee, Knoxville, TN, U.S.A.

V. A. Stanisich, La Trobe University, Bundoora, Australia

J. Stougaard, University of Aarhus, Aarhus, Denmark

M. Sutherland, University of Southern Queensland, Toowoomba, Australia

J. Sweigard, DuPont Agricultural Products, Newark, DE, U.S.A.

J. Takemoto, Utah State University. Logan, UT, U.S.A.

T. Todd, Kansas State University, Manhattan, KS, U.S.A.

P. Tornero, University of North Carolina, Chappel Hill, NC, U.S.A.
I. Toth, Scottish Crops Research Institute, Invergowrie, Dundee, Scotland, United Kingdom

F. Trail, Michigan State University, East Lansing, MI, U.S.A.

J. Turner, University of East Anglia, Norwich, United Kingdom

B. Tyler, University of California, Davis, CA, U.S.A.

M. Udvardi, Max Planck Institute for Molecular Plant Physiology, Golm, Germany

B. Valent, DuPont Agricultural Products, Wilmington, DE, U.S.A.

N. Van Alfen, Texas A\&M University, College Station, TX, U.S.A.

G. van den Ackerveken, Utrecht University. Utrecht, The Netherlands

J. van Kan, Wageningen Agricultural University, Wageningen, The Netherlands

L. C. van Loon, Utrecht University, Utrecht, The Netherlands

C. Vance, University of Minnesota, St. Paul, MN, U.S.A.

K. Vandenbosch, Texas A\&M University, College Station, TX, U.S.A.

J. Walton, Michigan State University, East Lansing, MI, U.S.A.

G.-Liang Wang, The Ohio State University, Columbus, OH, U.S. A.

X. Wang, Kansas State University, Manhattan, KS, U.S.A.

C. Webb, Kansas State University, Manhattan, KS, U.S.A.

D. A. Webster, Illinois Institute of Technology, Chicago, IL, U.S.A.

K.-M. Weltring, Westfalische Wilhelms-Universitat, Munster, Germany

V. Wiemken, University of Basel, Basel, Switzerland

P. Williams, University of Nottingham, Nottingham, United Kingdom

B. Williamson, Scottish Crop Research Institute, Invergowrie, Dundee, United Kingdom

M. Wilson Colorado College, Colorado Springs, CO, U.S.A.

W. Wintermantel, USDA-ARS, Salinas, CA, U.S.A.

S.-M. Wong, National University of Singapore, Singapore, Singapore

N. D. Young, Montana State University, Bozeman, MT, U.S.A.

J.-M. Zhou, Kansas State University, Manhattan, KS, U.S.A. 